\title{
Secondary Electron Generation by Low Energy Ion Beams
}

\author{
D C Joy ${ }^{* * *}$, Y Lin ${ }^{*}, \mathrm{H}$ H Meyers III ${ }^{* *}$, Hendrix Demers ${ }^{* *}$, D E Newbury ${ }^{* * *}$ \\ * E-Beam Facility, University of Tennessee, Knoxville, TN 37996-3840 \\ ** Oak Ridge National Laboratory, Oak Ridge TN 37831-60 \\ *** National Institute of Science and Technology, Gaithersburg, MD 20899
}

Low energy ion beams are being increasingly viewed as an alternative to, or even as a replacement for, low voltage SEMs. The beam interaction volumes in both cases are comparable in their size and their proximity to the sample surface, and both can produce high quality secondary electron images. However, although a cursory comparison of ion generated SE (iSE) and electron generated SE (eSE) images of the same area of a sample shows micrographs that can look very similar this is misleading because the nature of the iSE and eSE images are quite distinct. More experimental data and additional analysis of the beam interactions is therefore required if images are to be properly interpreted. The yield e of eSE, rises rapidly with incident beam energy E reaching a maximum value which is typically in the range 1.5-2 and occurs at an energy of a few hundred $\mathrm{eV}$ before then falling away as about $1 / \mathrm{E}$.. In the case of ion beam irradiation the kinetic production of iSE commences at a particle velocity of about $10^{7} \mathrm{~cm} / \mathrm{sec} 30 \mathrm{eV}$ for $\mathrm{He}$, (3keV for Ar) producing a yield i of iSE which rises almost linearly with the accelerating voltage and reaches typical values of $1.5-2.5$ for energies of the order of $20-30 \mathrm{kV}$. Thus while at low energies the eSE and iSE yields are comparable in magnitude, at higher energies the iSE yield is an order of magnitude or more larger. The iSE yield will eventually reach a maximum value and then begin to fall when once the interaction volume lies mostly below the escape depth of the SE. Both eSE and iSE yields also display a marked although apparently chaotic - dependence on the atomic number of the target (Z2) and, in the ion case, on the atomic number of the ion (Z1) itself. In the electron case the minima in the SE yield versus Z2 plot correspond to shell filling but there is presently insufficient evidence to confirm if the same is true for the ion SE case. Because the stopping powers of ion and electrons, and hence their range in a given material, are different it can be presumed that effects such as topographic contrast will be different in both magnitude and functional form although measured data about this is limited.

Experimental emitted electron spectra recorded from the same sample under electron and ion impact are very different. This is because a substantial component of the electron generated signal is produced by exiting backscattered electrons (BSE) and the energy spectrum of the emitted electrons therefore extends from zero up to the energy of the incident beam (figure 1). The electron spectra produced by low energy beams of light ions (e.g. He or Ar) by contrast cover only a very restricted energy range (Figure 2) because there is no backscattered electron component. However, significant ion backscattering does occur for most target materials at low energy so there will also be an 
backscattered ion induced "iSE2" contribution to the iSE signal although the relative magnitude of this component is not yet well characterized.

Electron and ions also induce very different charging behavior and this also has major implications for the SE images that they produce. For an electron beam impinging on an insulator which is initially electrically neutral (zero potential) the charge state depends on the sign of (1- eSE- eSE) where eSE and eSE are respectively the electron induced SE and BSE yields. As the beam energy is increased from zero this quantity is initially greater than zero implying negative charging; then between energies E1 and E2 charge deposition is positive; finally above E2 negative charging is reasserted. The low energy imaging of insulators in SE mode with an electron beam is therefore usually performed around the appropriate E2 energy for the material of interest (since E1 does not provide a stable operating condition). For an incident ion beam the incident particle has a positive charge, but both secondary electrons (negative) and charged backscattered ions (positive) are emitted. The charging during irradiation depends on the sign of $\left(1+\right.$ iSE $^{-}$ion $)$ where isE and ion are respectively the ion induced yields of secondary electrons and of charged backscattered ions. Since iSE is greater than zero and ion is always less than unity the magnitude of this expression is always greater than zero and so the charging will be positive in sign at all ion energies (i.e. there are no crossover points at which charging might be minimized). Ion generated secondary electron images will therefore always be strongly influenced by the recollection of SE that will occur because of the positive surface potential and surface charging will be a significant factor in determining the details of the iSE image unless a high field is applied to extract the SE efficiently.

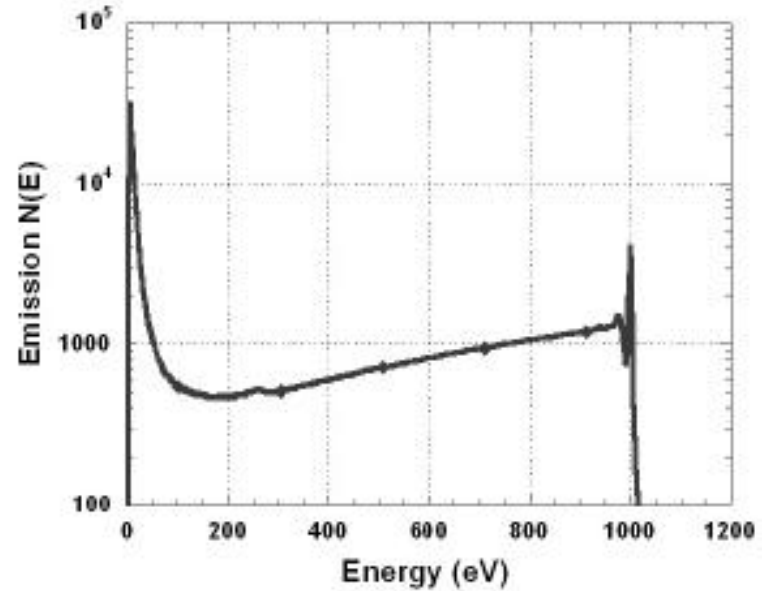

Fig1. Experimental electron spectrum from from iron irradiated by a $1 \mathrm{keV}$ electron beam.

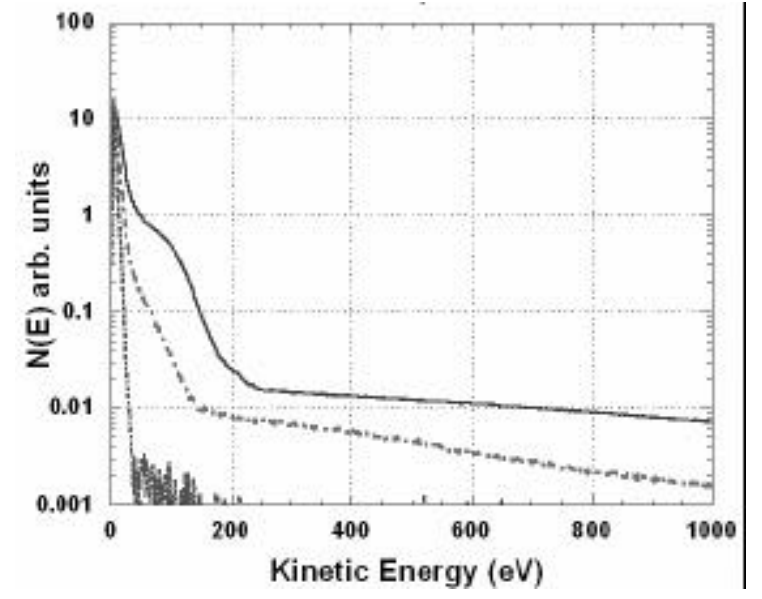

Fig.2 Experimental electron spectrum from iron irradiated by Ar+ beam with energies of $500 \mathrm{eV}, 1 \mathrm{keV}$, and $2 \mathrm{keV}$ 